\title{
3 Research Square

\section{Pack Years and Lower Lung Function is Associated With Ultra-short Telomeres in Copd: Evidence From Lung Tissue}

\section{Huseyin Cagsin}

Near East University

Nedime Serakinci ( $\nabla$ nedime.serakinci@neu.edu.tr)

Near East University https://orcid.org/0000-0002-1884-0839

Ali Uzan

Near East University

Finn Rasmussen

Near East University

\section{Research}

Keywords: Chronic obstructive pulmonary disease (COPD), bronchoalveolar, short telomeres, namely, ultra-short telomeres

Posted Date: June 24th, 2020

DOI: https://doi.org/10.21203/rs.3.rs-37099/v1

License: (c) (i) This work is licensed under a Creative Commons Attribution 4.0 International License. Read Full License 


\section{Abstract}

\section{Background}

Chronic obstructive pulmonary disease (COPD) is driven by a complicated mix of factors such as lifestyle and environmental exposures. Tobacco smoking is the main risk factor for forming chronic inflammation in COPD. Association between cigarette smoking and the role of telomere shortening in COPD has been studied mainly based on the assessment of mean telomere length on leukocytes instead of lung tissue where the primary damage occurs. Here we investigate this association in bronchoalveolar samples by using a new assay that specifically evaluates critically short telomeres, namely, ultra-short telomeres that have sizes less than $1.5 \mathrm{~kb}$.

\section{Methods}

The study was carried out on materials from the patients eligible for bronchoscopy as well as mild to severe persistent airway obstruction, defined as a post-bronchodilator ratio of less than $70 \%$. Bronchial washing (BW) and leukocyte samples were collected from 32 patients diagnosed with COPD. Telomere length evaluation was done with isolated DNA using Universal STELA to specifically identify the presence of ultra-short telomeres in samples. A t-Student, ANOVA, $\mathrm{Chi}^{2}$, and Paired Sample $\mathrm{T}$-test were used to test differences in means and proportions in statistical analysis. Two-tailed $p$-values $\leq 0.05$ were considered significant

\section{Results}

The location of BW did not show a significant difference when compared in terms of the presence of ultra-short telomeres $(p>0.05)$. Higher total pack-years was found amongst patients with ultra-short telomeres (32 packyears versus 16 packyears; $p=0.045)$, lower lung function (FEV1\%) $(51 \%$ versus $82 \%$; $\mathrm{p}<0.001)$ when compared with subjects with telomere length more than 1.5kbs in BW. An increasing number of total pack-years, older age and lower FEV1\% was observed through the groups comprising subjects with ultra-short telomeres in both BW and leukocytes, subjects with ultra-short telomeres only in BW and subjects with telomeres longer than $1.5 \mathrm{kbs}($ all $p<0.01)$

\section{Conclusions}

Our results emphasize the role of ultra-short telomeres in COPD, in vivo, especially when the lung tissue instead of leukocytes is investigated. Additionally, our results demonstrated a dose-response association between pack-years of smoking, low lung function, and ultra-short telomere length in COPD.

\section{Background}

Cigarette smoking has long been recognized as the leading cause of the development of chronic obstructive pulmonary disease (COPD). Destruction of the lung tissue (alveoli septae) with the development of emphysema is one of the cornerstones of COPD disease. The most widely recognized 
explanation for this phenomenon is that smoking activates inflammatory cells which with their proteolytic enzymes destroy alveoli septae [1]. This assumption is supported by the fact that individuals with a1-antitrypsin deficiency have an increased risk of developing emphysema at earlier ages [2], as these individuals have a lower level of enzymes that protect against degradation. Since the risk of developing COPD is also closely related to age, some pathogenesis of COPD could be a smoking-induced accelerated "aging" of the lung epithelial cells of particularly sensitive individuals with increased cell death, apoptosis [3]. Lung tissue fails to reconstruct itself after a gradually increased number of senescent cells. This may also be contributed by the short telomeres which promote cellular senescence [4]. Both apoptosis and senescence are triggered as a DNA damage response through the p53 pathway if the damage is beyond repair [5]. These DNA damage results from genotoxic effects from the environment. In lung epithelium, several genotoxic components are found in cigarette smoke, and in connection with studies of the etiology of lung cancer, the accumulation of DNA damage in lung cells in tobacco smokers has been demonstrated [6].

Tobacco smoking is the main risk factor for forming chronic inflammation in COPD. This inflammation gradually results in structural changes in the small airway, and thereby persistent airway obstruction through accelerated aging in lung tissue [7]. Aging, even in non-smoking subjects, is related to a decreasing forced expiratory volume in 1 second (FEV1)/ forced vital capacity (FVC) (FEV1/FVC) ratio which is a sign of increased airway limitation [8]. It is thought that chronic inflammation by other environmental exposures or cellular aging itself might be a reason for the airway limitation. The associations between telomeres and inflammatory diseases like COPD and aging-related diseases are often studied on leukocytes [9]. This might be one of the reasons that telomere length associations with COPD remain unclear.

COPD is mainly a disease in the lung. COPD is a progressive disease that seems to affect the body with many co-morbidities such as heart and musculoskeletal problems. The development of COPD affects humans very differently therefore individual's regenerative ability of lung tissue is important. a decrease in repair and regenerative ability likely leads to loss of lung cells and persistent airway obstruction [10]. A decrease in regenerative ability likely to be explained by telomere dysfunction which also takes place in normal aging and smoke-induced inflammations. There is also partly non-telomere-associated DNA damage that takes place [11-12].

COPD is driven by a complicated mix of factors such as lifestyle and environmental exposures that contribute to telomere shortening. Telomere length has been highlighted as a biomarker of aging and aging-related diseases. As aging takes place, telomeres gradually shorten because of cell division [13]. Furthermore, shorter telomeres have been identified as potential markers for several health problems in a wide range of diseases such as cardiovascular disease [14], neurodegenerative disorders [15], and cancers [16]. Often, telomere shortening is the biological effect of exogenous and hazardous exposures to chemicals like asbestos, smoking, and air pollution. Such exposures can cause DNA damage on the whole genome including telomeres which induces double-strand breaks in DNA thus increasing potential shortening of telomeres [17-18]. 
To date, there is no direct evidence of whether telomere length is a causal factor in mechanisms of how smoking affects lung function, particularly in patients diagnosed with COPD. In this study, we investigated the telomere length in lung epithelial cells that is isolated from bronchial washings (BW) that is related to smoking-induced COPD. In this connection, we group the study subject's samples according to clinical markers for disease stage (severity), tobacco use, and age.

\section{Methods}

\section{Collection of biological samples}

The hypothesis is tested on materials from the patients eligible for bronchoscopy as well as mild to severe persistent airway obstruction, defined as a post-bronchodilator ratio of less than $70 \%$. Lung function was expressed as a post-bronchodilator predicted value of forced expiratory volume in second (FEV1\%) according to the European Respiratory Society (ERS) [19]. This served also as a COPD grading 1 to 4 according to the Global Initiative for Chronic Obstructive Pulmonary Disease (GOLD) [20]. One packyear was defined as the average use of 20 cigarettes/day for one year. Patients were recruited from the pulmonary medicine Department at Erciyes University Hospital Bronchoscopy Unit. 32 subjects were included. 17 patients (53\%) were diagnosed with lung cancer, 1 patient had lung metastasis (3\%) and 14 patients (44\%) no abnormalities were found in the bronchoscopy. The recruitment of cells for telomere measurement was done at the end of the bronchoscopy. BW was done in the opposite bronchial tree than the routine samples in which sampled with a pre-wash and then applying $20 \mathrm{ml}$ isotonic $\mathrm{NaCl}$. Figure 1 shows where the sampling was done. A peripheral blood sample for leukocyte telomere length was also taken from the subjects after the sampling with the bronchoscopy.

\section{Telomere length evaluation}

DNA from both BW and leukocytes were extracted by commercial kits (RTA technologies). UniversalSTELA was carried out as described previously [20] with minor alterations. To summarize, $1 \mu \mathrm{g}$ DNA was digested in a $50 \mu \mathrm{l}$ reaction containing $1 \mu \mathrm{l}$ Msel, $0.5 \mu \mathrm{l} \mathrm{Ndel}$ and $5 \mu \mathrm{l}$ cutsmart buffer in 1 hour at $37^{\circ} \mathrm{C}$. Msel and Ndel were subsequently inactivated at $65^{\circ} \mathrm{C}$ in 20 minutes. $0.05 \mu \mathrm{g}$ of digested DNA is added to $3 \mu \mathrm{l}$ of 12 mer and 42 mer panhandles in $7 \mu$ l of volume. The reaction temperature is decreased from $65^{\circ} \mathrm{C}$ to $16^{\circ} \mathrm{C}$ in 49 minutes. 20 units of T4 DNA ligase with $1.5 \mu \mathrm{l}$ of T4 DNA ligase buffer were quickly added to the reaction and volume was increased to $15 \mu \mathrm{l}$ with $6 \mu \mathrm{ldH} 20$ at $16^{\circ} \mathrm{C}$. Reactions were incubated overnight. After incubation 20 units of T4 DNA ligase and $2.5 \mu$ telorette working solution was added with $1 \mu \mathrm{l}$ of T4 DNA ligase buffer and volume was increased to $25 \mu \mathrm{l}$ with $6 \mu \mathrm{ldH} 2 \mathrm{O}$. Reactions were incubated at $35^{\circ} \mathrm{C}$ overnight and were inactivated by 20 minutes incubation at $65^{\circ} \mathrm{C}$. This was then followed by a PCR reaction which was exact to the previously described [20]. Universal STELA amplicons were separated by gel electrophoresis on a $0.8 \%$ agarose gel at $70 \mathrm{~V}$ for 3 hours. The separated DNA was transferred to a positively charged nylon membrane (Amersham). DNA fragments in the blot were hybridized to the DIG-labeled telomeric probe overnight at room temperature and incubated with a DIGspecific antibody with AP fragments. Chemiluminescence was detected with CDP-Star (Roche). All 
experiments were triplicated. Ultra-short telomeres were defined as telomeres shorter than $1.5 \mathrm{~kb}$ as established previously. Telomeres that are not shorter than $1.5 \mathrm{~kb}$ were defined as not critically short telomeres.

\section{Statistics}

A t-Student, ANOVA, $\mathrm{Chi}^{2}$, and Paired Sample $T$-test were used to test differences in means and proportions. Normality was tested by visual inspection of curves and by homogeneity testing. Twotailed $p$-values $\leq 0.05$ were considered significant. Statistical analysis was performed with the SPSS, IBM Co software ver. 23 (IBM Inc., New York, USA).

\section{Results}

\section{The section of the lung in BW does not influence telomere length measurement}

To investigate whether the BW localization is significant in telomere length measurement, the different locations in the lung were compared with respect to the presence of ultra-short telomeres. The location of BW did not show a significant difference when compared in terms of the presence of ultra-short telomeres ( $p>0.05$ ) (Figure 1). The median age of the 32 subjects was 63 years, range [31 to 73 years]. The median total pack-years 25 (20cig/day/year) range [0-106] and the median FEV1\% was $47 \%$, range [19-86\%]. $24(75 \%)$ of 32 subjects were males.

The presence of ultra-short telomeres was found in 26 out of 32 (81\%) in BW samples whilst ultra-short telomeres were found only 3 out of $32(9 \%)$ of leukocytes samples of same subjects. There was no case with ultra-short telomeres that were observed only in leukocytes. 23 out of 32 (72\%) subjects showed presents of ultra-short telomeres only in the bronchial washing and 6 out of $32(19 \%)$ had telomeres longer than $1.5 \mathrm{kbs}$.

\section{Higher pack-years and lower lung function is associated with the presence of ultra-short telomeres in lung tissue}

COPD grade 1 was seen in 8 of the subjects (25\%), grade 2 in 7 (22\%), grade 3 was $14(44 \%)$ and grade 4 was $3(9 \%)$ of the 32 subjects, respectively. Older age was significantly associated with COPD severity $(p=0.005)$, increasing total pack-years was not significantly associated with COPD grade $(p=0.88)$.

Higher total pack-years was found amongst patients with ultra-short telomeres (32 packyears versus 16 packyears; $p=0.045)$, lower lung function (FEV1\%) (51\% versus $82 \% ; p<0.001)$ when compared with subjects with telomere length more than $1.5 \mathrm{kbs}$ in BW (figure 2a).

Patients with ultra-short telomeres were older when compared to patients with telomere length above $1.5 \mathrm{kbs}$ (63 years versus 49 years) in BW but this association did not reach formal statistical significance $(p=0.06)$. 
Samples from leukocytes, only age (72 years versus 59 years; $p<0.001)$ were significantly associated with ultra-short telomeres when compared to telomeres longer than $1.5 \mathrm{kbs}$. A similar result was found for total pack-years (77 years versus 24 years; $p=0.058$ ), and FEV1\% ( $37 \%$ versus $58 \% ; p=0.12$ ) between ultra-short telomeres in leukocytes and not critically short telomeres (figure $2 b$ ).

\section{Incidence of total pack-years, age, and lung function}

The incidence of total pack-years, age, and lung function (FEV1\%) was compared among three distinct groups; patients with ultra-short telomeres in both BW and leukocytes, patients with ultra-short telomeres only in BW and subjects with no critically short telomeres. An increasing number of total pack-years, older age and lower FEV1\% was observed through the groups comprising subjects with ultra-short telomeres in both BW and leukocytes, subjects with ultra-short telomeres only in BW and subjects with telomeres longer than 1.5kbs(all $\mathrm{p}<0.01$ ) (figure 3 ).

\section{Discussion}

To our knowledge, this is the first study reporting the association between ultra-short telomeres and smoking in lung tissue in patients diagnosed with COPD concerning total pack-years. The results of this study are not influenced by the location of BW. The results suggest a dose-response relationship between pack-years of smoking, low lung function in COPD, and ultra-short telomere length. This implies a link between ultra-short telomere length, total tobacco smoke exposure, presence of persistent airway limitation as well as aging-related disease.

Previous reports on associations between telomere length with smoking were with leukocytes [21], lung tissue from mice [22], and from people who were not diagnosed with COPD [23]. Here in this study, there were no associations between telomere length and total pack-years and lung function parameters in leukocytes in our study group. In addition to this, there were not any subjects who had ultra-short telomeres in leukocytes and lack in lung tissue, whereas $72 \%$ of the COPD subjects only had ultra- short telomere in the lung tissue. Our results indicate that measuring ultra-short telomeres in lung tissue may detect smoking-related lung damage earlier than in leukocytes. Our finding suggests that abrupt telomere shortening is an important DNA damage indicator of the effects of cigarette smoke on lung tissue from patients diagnosed with COPD. Thus, the evaluation of telomere length in patients diagnosed with COPD is strongly suggested to be carried out in the lung tissue.

We confirmed previous findings that telomere length was shorter with older age in the leukocytes of the patients diagnosed with COPD [22,24-25]. As natural or accelerated aging occurs in the lungs, telomeres are expected to be shorter [22]. COPD risk factors contribute to cellular aging and result in reduced telomere length. The wide range of age in patients involved in the current study increases the representativeness of the results.

In a previous study, a sample of more than 45000 individuals, telomere length was moderately associated with FEV1 in leukocytes [26]. This is also the case when other pulmonary diseases are included [27] 
although telomere length and FEV1 association is stronger in COPD. Albeit a significantly smaller sample size, here we report a significant association between ultra-short telomeres and FEV1 in the lung tissue. This may be attributed to the lung being the site of injury in COPD may increase the likelihood of having ultra-short telomeres.

The main limitation of this study was the small sample size. Thus, sex-specific effects were not possible to be evaluated. Due to our cross-sectional approach, longitudinal effects between telomere length, total pack-years, and lung function cannot be studied.

\section{Conclusions}

We conclude that ultra-short telomeres are associated with increased pack-years, age, and decreased lung function in patients diagnosed with COPD irrespective of BW location. Pack-years is an important variable in telomere shortening and thus lower lung function in COPD. The effects of smoking can be determined at an earlier stage if the telomere evaluation is carried out in the lung tissue.

\section{Abbreviations}

1. Chronic obstructive pulmonary disease- COPD

2. Bronchial washing- BW

3. Forced expiratory volume in 1 second- FEV1

4. Forced vital capacity - FVC

5. predicted value of forced expiratory volume in second- FEV1\%

6. European Respiratory Society- ERS

7. Global Initiative for Chronic Obstructive Pulmonary Disease - GOLD

\section{Declarations}

\section{Ethics approval and consent to participate}

Near East University granted Ethical approval to carry out the study within its facilities (Ethical Application Ref: YDU-2015/30-202) with informed consent from participants.

\section{Consent for publication}

Not applicable

\section{Availability of data and materials}

The datasets used and/or analysed during the current study are available from the corresponding author on reasonable request. 


\section{Competing interest}

The authors declare that they have no competing interest

\section{Funding}

Region Syddanmarks Forskningspulje, "Telomerforkortning og DNA-skader i lungevæv ved KOL"

Region Syddanmarks Forskningspulje, "Telomere shortening in lung epithelial cells in patients with chronic obstructive pulmonary disease (COPD)"Authors' contributions

\section{Authors' contributions}

$\mathrm{HC}$ performed the laboratory work for telomere length evaluation, contribute to generation of raw data, interpretation of data and writing of manuscript. NS supervised the study, contribute to study design, telomere length evaluation, interpretation of data and writing and reviewing of this manuscript. AU collected patient samples and clinical information of patients. FR is a contribute to study design, evaluate the patient's clinical data, carried out the statistical analysis and contribute to interpretation of result and writing and reviewing the manuscript.

\section{Acknowledgments}

Not Applicable

\section{References}

1. Olloquequi J, Jaime S, Parra V, Cornejo-Cordova E, Valdivia G, Agusti A, et al. Comparative analysis of COPD associated with tobacco smoking, biomass smoke exposure or both. Respir Res. 2018; 30;19:77.

2. Laurell CB, Eriksson S.The electrophoretic a1-globulin pattern of serum in a1-antitrypsin deficiency. 2013; 10: Suppl. 1: 3-8

3. Vij N, Chandramani-Shivalingappa P, Van Westphal C, Hole R, Bodas M. Cigarette smoke-induced autophagy impairment accelerates lung aging, COPD-emphysema exacerbations and pathogenesis. Am J Physiol Cell Physiol. 2018; 314: 73-

4. Alder JK, Guo N, Kembou F, Perry EM, Anderson CJ, Gorgy Al, et al. Telomere length is a determinant of emphysema susceptibility. Am J Respir Crit Care Med. 2011; 184: 904-

5. Ou HL, Schumacher B. DNA damage responses and p53 in the aging process. Blood. 2018; 131: 488-

6. Pfeifer GP, Denissenko MF, Olivier M, Tretyakova N, Hecht SS, Hainaut P. Tobacco smoke carcinogens, DNA damage and p53 mutations in smoking-associated cancers. Oncogene. 2002; 21: 7435-

7. Agarwal AR, Kadam S, Brahme A, Agrawal M, Apte K, Narke G, et al. Systemic Immuno-metabolic alterations in chronic obstructive pulmonary disease (COPD). Respir Res. 2019; 20:171. 
8. Sexton P, Black P, Wu L, Sommerville F, Hamed M, Milne D, et al. Chronic obstructive pulmonary disease in non-smokers: a case-comparison study. COPD. 2014; 11: 2-

9. Mui TSY, Man JM, McElhaney JE, Sandford AJ, Coxson HO, Birmingham CL, et al. Telomere length and chronic obstructive pulmonary disease: evidence of accelerated aging. J Am Geriatr Soc. 2009; 57: 2372-2374.

10. Postma DS, Bush A, Van den Berge M. Risk factors and early origins of chronic obstructive pulmonary disease. Lancet. 2015; 385: 899-909.

11. Aoshiba K, Nagai A. Senescence hypothesis for the pathogenetic mechanism of chronic obstructive pulmonary disease. Proc Am Thorac Soc. 2009; 6: 596-601.

12. Aoshiba K, Zhou F, Tsuji T, Nagai A. DNA damage as a molecular link in the pathogenesis of COPD in smokers. Eur Respir J. 2012; 39: 1368-1376.

13. Aubert G, Lansdorp PM. Telomeres and aging. Physiol Rev. 2008; 88: 557-

14. Hammadah M, Al Mheid I, Wilmot K, Ramadan R, Abdelhadi N, Alkhoder A, et al. Telomere Shortening, Regenerative Capacity, and Cardiovascular Outcomes. Circ Res. 2017; 120: 1130-

15. Eitan E, Hutchison ER, Mattson MP. Telomere shortening in neurological disorders: an abundance of unanswered questions. Trends Neurosci. 2014; 37: 256-

16. Barthel FP, Wei W, Tang M, Martinez-Ledesma E, Hu X, Amin SB, et al. Systematic analysis of telomere length and somatic alterations in 31 cancer types. Nature Genet. 2017; 49: 349-357.

17. McDonough JE, Martens DS, Tanabe N, Ahangari F, Verleden SE, Maes K, et al. A role for telomere length and chromosomal damage in idiopathic pulmonary fibrosis. Respir Res. 2018; 19: 132.

18. Valavanidis A, Vlachogianni T, Fiotakis K, Loridas S. Pulmonary oxidative stress, inflammation and cancer: respirable particulate matter, fibrous dusts and ozone as major causes of lung carcinogenesis through reactive oxygen species mechanisms. Int J Environ Res Public Health. 2013; 10: 3886-

19. Viegi G, Pedreschi M, Pistelli F, Dede FD, Baldacci S, Carrozzi S, et al. Prevalence of airways obstruction in a general population: European Respiratory Society vs American Thoracic Society definition. Chest. 2000; 117 Suppl 2: 339S-

20. Karloh M, Mayer AF, Maurici R, Pizzichini MMM, Jones PW, Pizzichini E. The COPD Assessment Test: What Do We Know So Far?: A Systematic Review and Meta-Analysis About Clinical Outcomes Prediction and Classification of Patients Into GOLD Stages. Chest. 2016; 149: 413-425.

21. Bendix L, Horn PB, Jensen UB, Rubelj I, Kolvraa S. The load of short telomeres, estimated by a new method, Universal STELA, correlates with number of senescent cells. Aging Cell. 2010; 9: 383-397.

22. Savale L, Chaouat A, Bastuji-Garin S, Marcos E, Boyer L, Maitre B, et al. Shortened telomeres in circulating leukocytes of patients with chronic obstructive pulmonary disease. Am J Respir Crit Care Med. 2009; 179: 566-

23. Birch J, Anderson RK, Correia-Melo C, Jurk D, Hewit G, Marques FM, et al. DNA damage response at telomeres contributes to lung aging and chronic obstructive pulmonary disease. Am J Physiol Lung 
Cell Mol Physiol. 2015; 309: L1124-

24. Walters MS, De BP, Salit J, Buro-Auriemma LJ, Wilson T, Rogalski AM, et al. Smoking accelerates aging of the small airway epithelium. Respir Res. 2014; 15: 94.

25. Houben JM, Mercken EM, Ketelslegers HB, et al. Telomere shortening in chronic obstructive pulmonary disease. Respir Med. 2009;103(2):230-

26. Rode L, Bojesen SE, Weischer M, Vestbo J, Nordestgaard BG.Short telomere length, lung function and chronic obstructive pulmonary disease in 46,396 individuals. 2013; 68: 429-435.

27. Albrecht E, Sillanpää E, Karrasch S, Alves AC, Codd V, Hovatta I et al. Telomere length in circulating leukocytes is associated with lung function and disease. Eur Respir J. 2014; 43: 983-992.

\section{Figures}
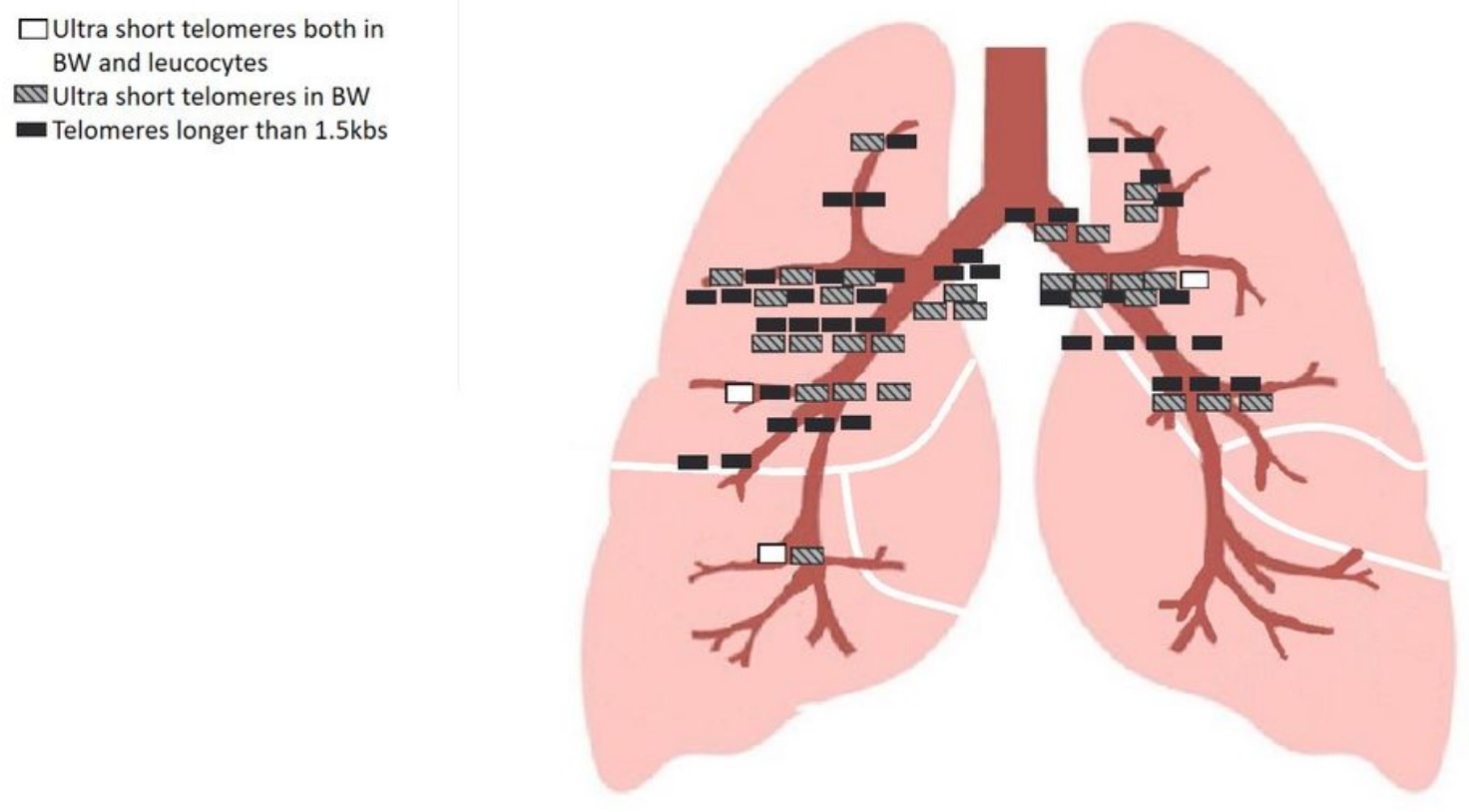

\section{Figure 1}

Bronchial tree showing the different locations of the bronchial washing sample (BW) location Presence of ultra-short telomeres in both BW and leukocytes (white bars), ultra-short telomeres only in BW (shade bars), and only telomeres longer than $1.5 \mathrm{kbs}$ (black bars). 
Figure 2
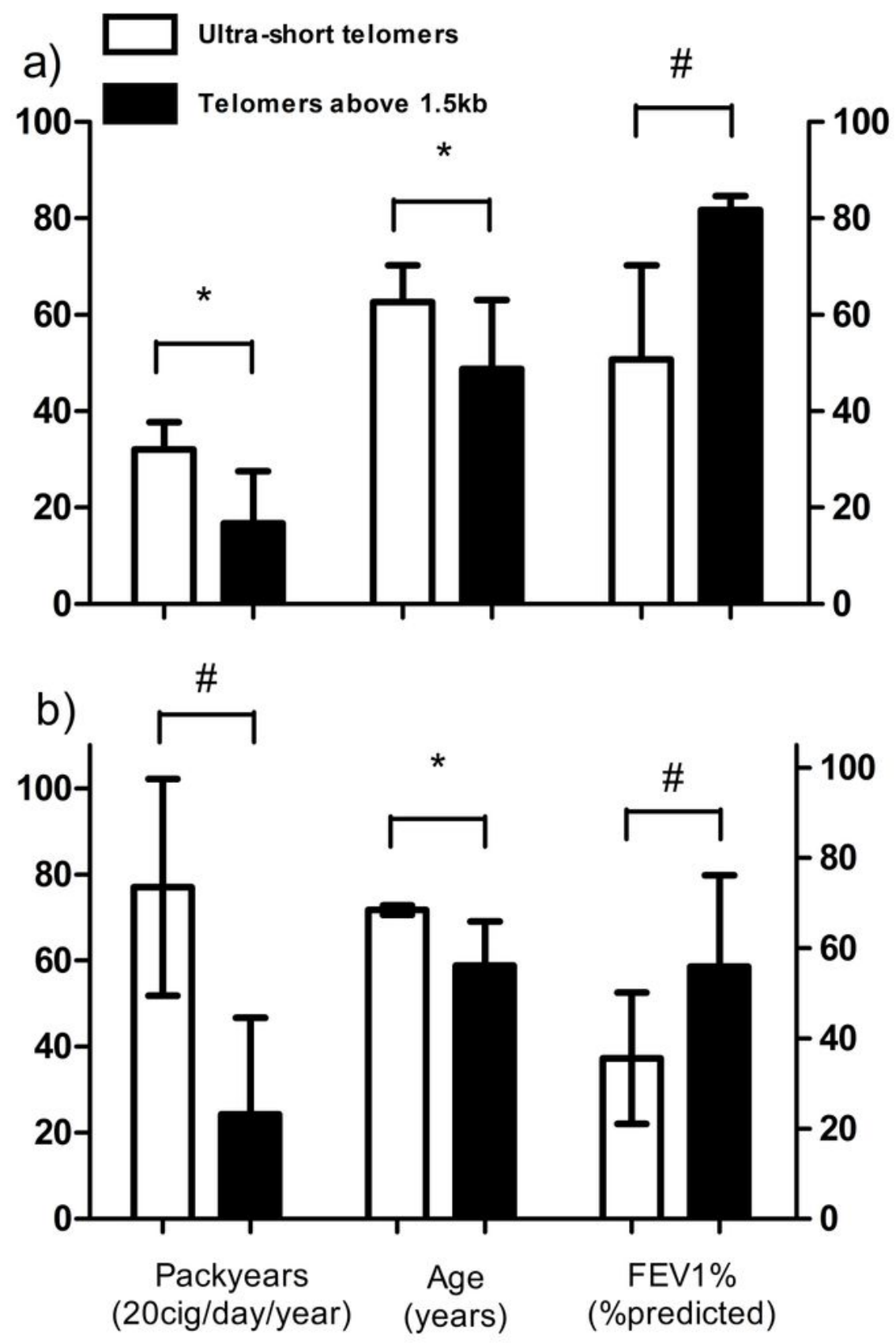

Figure 2

Distribution of total pack-years, age and lung function with respect to ultra-short telomeres Ultra-short telomeres (white bars) and telomeres longer than 1.5kbs (black bars) in bronchial washing samples (a) and leukocyte samples (b). ${ }^{*}=p<0.05, \#=p>0.05, \mathrm{FEV} 1 \%=$ predicted value of Forced Expiratory Volume in 1 second. 


\section{Figure 3}

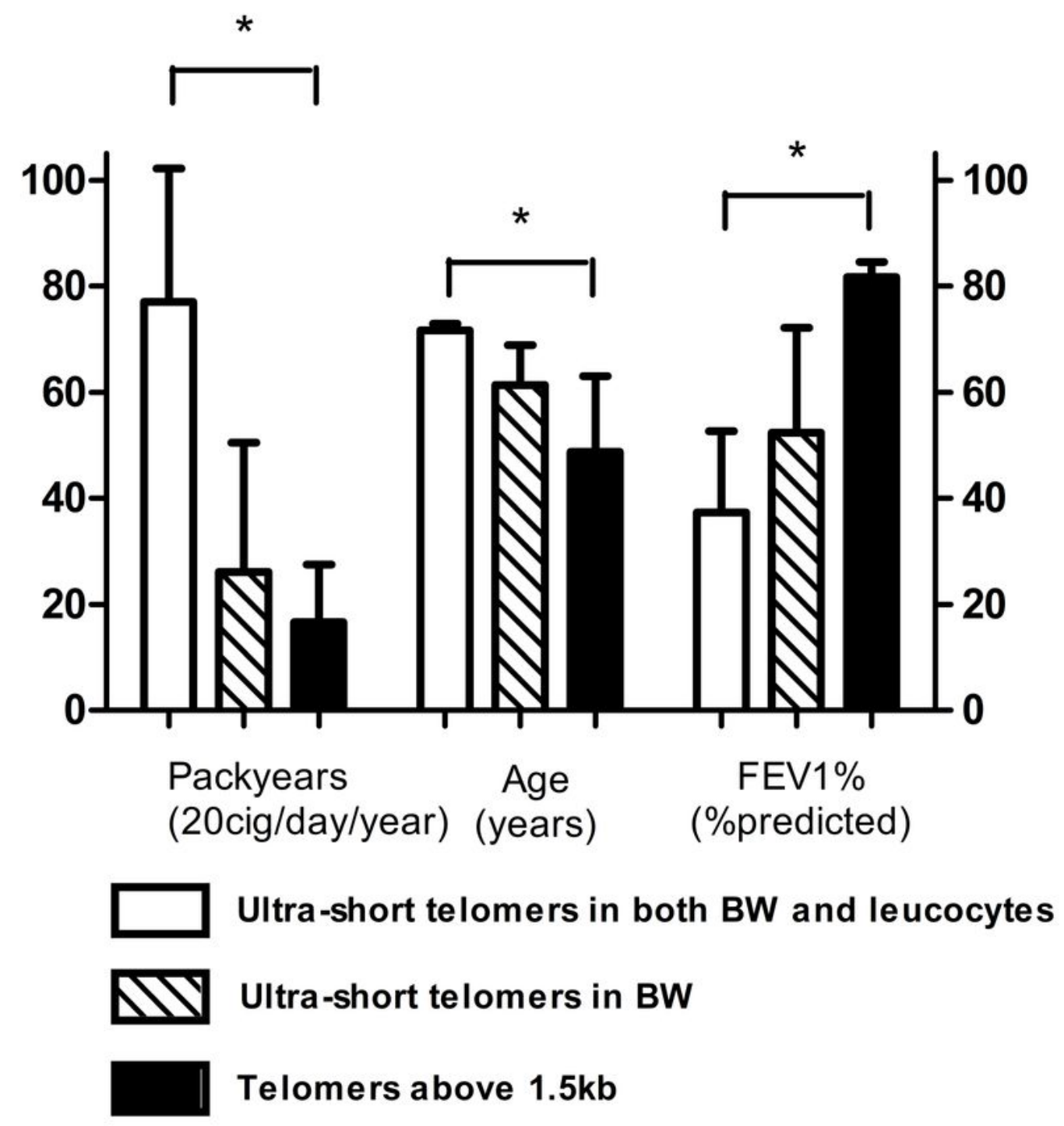

Figure 3

Incidence comparison of total pack-years, age, and lung function Subjects with ultra-short telomeres compared to subjects with telomeres longer than $1.5 \mathrm{kbs}$ in both bronchial washing samples and leukocyte samples(white bars); subjects with ultra-short telomeres only in bronchial washing samples (shaded bars) and subjects with telomeres longer than $1.5 \mathrm{kbs}$ (black bars). ${ }^{*}=p<0.05, \mathrm{FEV} 1 \%=$ predicted value of Forced Expiratory Volume in 1 second. 